\title{
VERMA MODULES AND PREPROJECTIVE ALGEBRAS
}

\author{
CHRISTOF GEISS*, BERNARD LECLERC ${ }^{\dagger}$ AND \\ JAN SCHRÖER
}

\section{Dedicated to George Lusztig on the occasion of his sixtieth birthday}

\begin{abstract}
We give a geometric construction of the Verma modules of a symmetric Kac-Moody Lie algebra $\mathfrak{g}$ in terms of constructible functions on the varieties of nilpotent finite-dimensional modules of the corresponding preprojective algebra $\Lambda$.
\end{abstract}

\section{$\S 1$. Introduction}

Let $\mathfrak{g}$ be the symmetric Kac-Moody Lie algebra associated to a finite unoriented graph $\Gamma$ without loop. Let $\mathfrak{n}_{-}$denote a maximal nilpotent subalgebra of $\mathfrak{g}$. In [Lu1, §12], Lusztig has given a geometric construction of $U\left(\mathfrak{n}_{-}\right)$in terms of certain Lagrangian varieties. These varieties can be interpreted as module varieties for the preprojective algebra $\Lambda$ attached to the graph $\Gamma$ by Gelfand and Ponomarev [GP]. In Lusztig's construction, $U\left(\mathfrak{n}_{-}\right)$ gets identified with an algebra $(\mathcal{M}, *)$ of constructible functions on these varieties, where $*$ is a convolution product inspired by Ringel's multiplication for Hall algebras.

Later, Nakajima gave a similar construction of the highest weight irreducible integrable $\mathfrak{g}$-modules $L(\lambda)$ in terms of some new Lagrangian varieties which differ from Lusztig's ones by the introduction of some extra vector spaces $W_{k}$ for each vertex $k$ of $\Gamma$, and by considering only stable points instead of the whole variety $[\mathrm{Na}, \S 10]$.

Received November 18, 2004.

2000 Mathematics Subject Classification: 14M99, 16G20, 17B35, $17 \mathrm{~B} 67$.

${ }^{*}$ C. Geiss acknowledges support from DGAPA grant IN101402-3.

${ }^{\dagger}$ B. Leclerc is grateful to the GDR 2432 and the GDR 2249 for their support.

${ }^{\ddagger}$ J. Schröer was supported by a research fellowship from the DFG (Deutsche Forschungsgemeinschaft). 
The aim of this paper is to extend Lusztig's original construction and to endow $\mathcal{M}$ with the structure of a Verma module $M(\lambda)$.

To do this we first give a variant of the geometrical construction of the integrable $\mathfrak{g}$-modules $L(\lambda)$, using functions on some natural open subvarieties of Lusztig's varieties instead of functions on Nakajima's varieties (Theorem 1). These varieties have a simple description in terms of the preprojective algebra $\Lambda$ and of certain injective $\Lambda$-modules $q_{\lambda}$.

Having realized the integrable modules $L(\lambda)$ as quotients of $\mathcal{M}$, it is possible, using the comultiplication of $U\left(\mathfrak{n}_{-}\right)$, to construct geometrically the raising operators $E_{i}^{\lambda} \in \operatorname{End}(\mathcal{M})$ which make $\mathcal{M}$ into the Verma module $M(\lambda)$ (Theorem 2). Note that we manage in this way to realize Verma modules with arbitrary highest weight (not necessarily dominant).

Finally, we dualize this setting and give a geometric construction of the dual Verma module $M(\lambda)^{*}$ in terms of the delta functions $\delta_{x} \in \mathcal{M}^{*}$ attached to the finite-dimensional nilpotent $\Lambda$-modules $x$ (Theorem 3 ).

\section{$\S 2$. Verma modules}

2.1. Let $\mathfrak{g}$ be the symmetric Kac-Moody Lie algebra associated with a finite unoriented graph $\Gamma$ without loop. The set of vertices of the graph is denoted by $I$. The (generalized) Cartan matrix of $\mathfrak{g}$ is $A=\left(a_{i j}\right)_{i, j \in I}$, where $a_{i i}=2$ and, for $i \neq j,-a_{i j}$ is the number of edges between $i$ and $j$.

2.2. Let $\mathfrak{g}=\mathfrak{n} \oplus \mathfrak{h} \oplus \mathfrak{n}_{-}$be a Cartan decomposition of $\mathfrak{g}$, where $\mathfrak{h}$ is a Cartan subalgebra and $\left(\mathfrak{n}, \mathfrak{n}_{-}\right)$a pair of opposite maximal nilpotent subalgebras. Let $\mathfrak{b}=\mathfrak{n} \oplus \mathfrak{h}$. The Chevalley generators of $\mathfrak{n}\left(\right.$ resp. $\left.\mathfrak{n}_{-}\right)$are denoted by $e_{i}(i \in I)\left(\right.$ resp. $\left.f_{i}\right)$ and we set $h_{i}=\left[e_{i}, f_{i}\right]$.

2.3. Let $\alpha_{i}$ denote the simple root of $\mathfrak{g}$ associated with $i \in I$. Let $(-;-)$ be a symmetric bilinear form on $\mathfrak{h}^{*}$ such that $\left(\alpha_{i} ; \alpha_{j}\right)=a_{i j}$. The lattice of integral weights in $\mathfrak{h}^{*}$ is denoted by $P$, and the sublattice spanned by the simple roots is denoted by $Q$. We put

$$
P_{+}=\left\{\lambda \in P \mid\left(\lambda ; \alpha_{i}\right) \geqslant 0,(i \in I)\right\}, \quad Q_{+}=Q \cap P_{+} .
$$

2.4. Let $\lambda \in P$ and let $M(\lambda)$ be the Verma module with highest weight $\lambda$. This is the induced $\mathfrak{g}$-module defined by $M(\lambda)=U(\mathfrak{g}) \otimes_{U(\mathfrak{b})} \mathbb{C} u_{\lambda}$, where $u_{\lambda}$ is a basis of the one-dimensional representation of $\mathfrak{b}$ given by

$$
h u_{\lambda}=\lambda(h) u_{\lambda}, \quad n u_{\lambda}=0, \quad(h \in \mathfrak{h}, n \in \mathfrak{n}) .
$$


As a $P$-graded vector space $M(\lambda) \cong U\left(\mathfrak{n}_{-}\right)$(up to a degree shift by $\lambda$ ). $M(\lambda)$ has a unique simple quotient denoted by $L(\lambda)$, which is integrable if and only if $\lambda \in P_{+}$. In this case, the kernel of the $\mathfrak{g}$-homomorphism $M(\lambda) \rightarrow L(\lambda)$ is the $\mathfrak{g}$-module $I(\lambda)$ generated by the vectors

$$
f_{i}^{\left(\lambda ; \alpha_{i}\right)+1} \otimes u_{\lambda}, \quad(i \in I) .
$$

\section{$\S 3$. Constructible functions}

3.1. Let $X$ be an algebraic variety over $\mathbb{C}$ endowed with its Zariski topology. A map $f$ from $X$ to a vector space $V$ is said to be constructible if its image $f(X)$ is finite, and for each $v \in f(X)$ the preimage $f^{-1}(v)$ is a constructible subset of $X$.

3.2. By $\chi(A)$ we denote the Euler characteristic of a constructible subset $A$ of $X$. For a constructible map $f: X \rightarrow V$ one defines

$$
\int_{x \in X} f(x)=\sum_{v \in V} \chi\left(f^{-1}(v)\right) v \in V .
$$

More generally, for a constructible subset $A$ of $X$ we write

$$
\int_{x \in A} f(x)=\sum_{v \in V} \chi\left(f^{-1}(v) \cap A\right) v .
$$

\section{$\S 4$. Preprojective algebras}

4.1. Let $\Lambda$ be the preprojective algebra associated to the graph $\Gamma$ (see for example [Ri], [GLS]). This is an associative $\mathbb{C}$-algebra, which is finitedimensional if and only if $\Gamma$ is a graph of type $A, D, E$. Let $s_{i}$ denote the simple one-dimensional $\Lambda$-module associated with $i \in I$, and let $p_{i}$ be its projective cover and $q_{i}$ its injective hull. Again, $p_{i}$ and $q_{i}$ are finitedimensional if and only if $\Gamma$ is a graph of type $A, D, E$.

4.2. A finite-dimensional $\Lambda$-module $x$ is nilpotent if and only if it has a composition series with all factors of the form $s_{i}(i \in I)$. We will identify the dimension vector of $x$ with an element $\beta \in Q_{+}$by setting $\operatorname{dim}\left(s_{i}\right)=\alpha_{i}$.

4.3. Let $q$ be an injective $\Lambda$-module of the form

$$
q=\bigoplus_{i \in I} q_{i}^{\oplus a_{i}}
$$

for some nonnegative integers $a_{i}(i \in I)$. 
LEMMA 1. Let $x$ be a finite-dimensional $\Lambda$-module isomorphic to a submodule of $q$. If $f_{1}: x \rightarrow q$ and $f_{2}: x \rightarrow q$ are two monomorphisms, then there exists an automorphism $g: q \rightarrow q$ such that $f_{2}=g f_{1}$.

Proof. Indeed, $q$ is the injective hull of its socle $b=\bigoplus_{i \in I} s_{i}^{\oplus a_{i}}$. Let $c_{j}$ $(j=1,2)$ be a complement of $f_{j}(\operatorname{socle}(x))$ in $b$. Then $c_{1} \cong c_{2}$ and the maps

$$
h_{j}:=f_{j} \oplus \mathrm{id}: \quad x \oplus c_{j} \longrightarrow q, \quad(j=1,2)
$$

are injective hulls. The result then follows from the unicity of the injective hull.

Hence, up to isomorphism, there is a unique way to embed $x$ into $q$.

4.4. Let $\mathcal{M}$ be the algebra of constructible functions on the varieties of finite-dimensional nilpotent $\Lambda$-modules defined by Lusztig [Lu2] to give a geometric realization of $U\left(\mathfrak{n}_{-}\right)$. We recall its definition.

For $\beta=\sum_{i \in I} b_{i} \alpha_{i} \in Q_{+}$, let $\Lambda_{\beta}$ denote the variety of nilpotent $\Lambda$ modules with dimension vector $\beta$. Recall that $\Lambda_{\beta}$ is endowed with an action of the algebraic group $G_{\beta}=\prod_{i \in I} G L_{b_{i}}(\mathbb{C})$, so that two points of $\Lambda_{\beta}$ are isomorphic as $\Lambda$-modules if and only if they belong to the same $G_{\beta}$-orbit. Let $\widetilde{\mathcal{M}}_{\beta}$ denote the vector space of constructible functions from $\Lambda_{\beta}$ to $\mathbb{C}$ which are constant on $G_{\beta}$-orbits. Let

$$
\widetilde{\mathcal{M}}=\bigoplus_{\beta \in Q_{+}} \widetilde{\mathcal{M}}_{\beta}
$$

One defines a multiplication $*$ on $\widetilde{\mathcal{M}}$ as follows. For $f \in \widetilde{\mathcal{M}}_{\beta}, g \in \widetilde{\mathcal{M}}_{\gamma}$ and $x \in \Lambda_{\beta+\gamma}$, we have

$$
(f * g)(x)=\int_{U} f\left(x^{\prime}\right) g\left(x^{\prime \prime}\right),
$$

where the integral is over the variety of $x$-stable subspaces $U$ of $x$ of dimension $\gamma, x^{\prime \prime}$ is the $\Lambda$-submodule of $x$ obtained by restriction to $U$ and $x^{\prime}=x / x^{\prime \prime}$. In the sequel in order to simplify notation, we will not distinguish between the subspace $U$ and the submodule $x^{\prime \prime}$ of $x$ carried by $U$. Thus we shall rather write

$$
(f * g)(x)=\int_{x^{\prime \prime}} f\left(x / x^{\prime \prime}\right) g\left(x^{\prime \prime}\right),
$$


where the integral is over the variety of submodules $x^{\prime \prime}$ of $x$ of dimension $\gamma$.

For $i \in I$, the variety $\Lambda_{\alpha_{i}}$ is reduced to a single point : the simple module $s_{i}$. Denote by $\mathbf{1}_{i}$ the function mapping this point to 1 . Let $\mathcal{G}(i, x)$ denote the variety of all submodules $y$ of $x$ such that $x / y \cong s_{i}$. Then by (2) we have

$$
\left(\mathbf{1}_{i} * g\right)(x)=\int_{y \in \mathcal{G}(i, x)} g(y) .
$$

Let $\mathcal{M}$ denote the subalgebra of $\widetilde{\mathcal{M}}$ generated by the functions $\mathbf{1}_{i}(i \in$ $I)$. By Lusztig [Lu2], $(\mathcal{M}, *)$ is isomorphic to $U\left(\mathfrak{n}_{-}\right)$by mapping $\mathbf{1}_{i}$ to the Chevalley generator $f_{i}$.

4.5. In the identification of $U\left(\mathfrak{n}_{-}\right)$with $\mathcal{M}$, formula (3) represents the left multiplication by $f_{i}$. In order to endow $\mathcal{M}$ with the structure of a Verma module we need to introduce the following important definition. For $\nu \in P_{+}$, let

$$
q_{\nu}=\bigoplus_{i \in I} q_{i}^{\oplus\left(\nu ; \alpha_{i}\right)} .
$$

Lusztig has shown [Lu3, §2.1] that Nakajima's Lagrangian varieties for the geometric realization of $L(\nu)$ are isomorphic to the Grassmann varieties of $\Lambda$-submodules of $q_{\nu}$ with a given dimension vector.

Let $x$ be a finite-dimensional nilpotent $\Lambda$-module isomorphic to a submodule of the injective module $q_{\nu}$. Let us fix an embedding $F: x \rightarrow q_{\nu}$ and identify $x$ with a submodule of $q_{\nu}$ via $F$.

Definition 1. For $i \in I$ let $\mathcal{G}(x, \nu, i)$ be the variety of submodules $y$ of $q_{\nu}$ containing $x$ and such that $y / x$ is isomorphic to $s_{i}$.

This is a projective variety which, by 4.3, depends only (up to isomorphism) on $i, \nu$ and the isoclass of $x$.

\section{$\S 5$. Geometric realization of integrable irreducible $\mathfrak{g}$-modules}

5.1. For $\lambda \in P_{+}$and $\beta \in Q_{+}$, let $\Lambda_{\beta}^{\lambda}$ denote the variety of nilpotent $\Lambda$-modules of dimension vector $\beta$ which are isomorphic to a submodule of $q_{\lambda}$. Equivalently $\Lambda_{\beta}^{\lambda}$ consists of the nilpotent modules of dimension vector $\beta$ whose socle contains $s_{i}$ with multiplicity at most $\left(\lambda ; \alpha_{i}\right)(i \in I)$. This variety has been considered by Lusztig [Lu4, $\S 1.5]$. In particular it is known that $\Lambda_{\beta}^{\lambda}$ is an open subset of $\Lambda_{\beta}$, and that the number of its irreducible components is equal to the dimension of the $(\lambda-\beta)$-weight space of $L(\lambda)$. 
5.2. Define $\widetilde{\mathcal{M}}_{\beta}^{\lambda}$ to be the vector space of constructible functions on $\Lambda_{\beta}^{\lambda}$ which are constant on $G_{\beta}$-orbits. Let $\mathcal{M}_{\beta}^{\lambda}$ denote the subspace of $\widetilde{\mathcal{M}}_{\beta}^{\lambda}$ obtained by restricting elements of $\mathcal{M}_{\beta}$ to $\Lambda_{\beta}^{\lambda}$. Put $\widetilde{\mathcal{M}}^{\lambda}=\bigoplus_{\beta} \widetilde{\mathcal{M}}_{\beta}^{\lambda}$ and $\mathcal{M}^{\lambda}=\bigoplus_{\beta} \mathcal{M}_{\beta}^{\lambda}$. For $i \in I$ define endomorphisms $E_{i}, F_{i}, H_{i}$ of $\widetilde{\mathcal{M}}^{\lambda}$ as follows:

$$
\begin{array}{ll}
\left(E_{i} f\right)(x)=\int_{y \in \mathcal{G}(x, \lambda, i)} f(y), & \left(f \in \widetilde{\mathcal{M}}_{\beta}^{\lambda}, x \in \Lambda_{\beta-\alpha_{i}}^{\lambda}\right), \\
\left(F_{i} f\right)(x) & =\int_{y \in \mathcal{G}(i, x)} f(y), \quad\left(f \in \widetilde{\mathcal{M}}_{\beta}^{\lambda}, x \in \Lambda_{\beta+\alpha_{i}}^{\lambda}\right), \\
\left(H_{i} f\right)(x) & =\left(\lambda-\beta ; \alpha_{i}\right) f(x), \quad\left(f \in \widetilde{\mathcal{M}}_{\beta}^{\lambda}, x \in \Lambda_{\beta}^{\lambda}\right) .
\end{array}
$$

THEOREM 1. The endomorphisms $E_{i}, F_{i}, H_{i}$ of $\widetilde{\mathcal{M}}^{\lambda}$ leave stable the subspace $\mathcal{M}^{\lambda}$. Denote again by $E_{i}, F_{i}, H_{i}$ the induced endomorphisms of $\mathcal{M}^{\lambda}$. Then the assignments $e_{i} \mapsto E_{i}, f_{i} \mapsto F_{i}, h_{i} \mapsto H_{i}$, give a representation of $\mathfrak{g}$ on $\mathcal{M}^{\lambda}$ isomorphic to the irreducible representation $L(\lambda)$.

5.3. The proof of Theorem 1 will involve a series of lemmas.

5.3.1. For $\mathbf{i}=\left(i_{1}, \ldots, i_{r}\right) \in I^{r}$ and $\mathbf{a}=\left(a_{1}, \ldots, a_{r}\right) \in \mathbb{N}^{r}$, define the variety $\mathcal{G}(x, \lambda,(\mathbf{i}, \mathbf{a}))$ of flags of $\Lambda$-modules

$$
\mathfrak{f}=\left(x=y_{0} \subset y_{1} \subset \cdots \subset y_{r} \subset q_{\lambda}\right)
$$

with $y_{k} / y_{k-1} \cong s_{i_{k}}^{\oplus a_{k}}(1 \leqslant k \leqslant r)$. As in Definition 1 , this is a projective variety depending (up to isomorphism) only on (i,a), $\lambda$ and the isoclass of $x$ and not on the choice of a specific embedding of $x$ into $q_{\lambda}$.

Lemma 2. Let $f \in \widetilde{\mathcal{M}}_{\beta}^{\lambda}$ and $x \in \Lambda_{\beta-a_{1} \alpha_{i_{1}}-\cdots-a_{r} \alpha_{i_{r}}}^{\lambda}$. Put $E_{i}^{(a)}=$ $(1 / a !) E_{i}^{a}$. We have

$$
\left(E_{i_{r}}^{\left(a_{r}\right)} \cdots E_{i_{1}}^{\left(a_{1}\right)} f\right)(x)=\int_{\mathfrak{f} \in \mathcal{G}(x, \lambda,(\mathbf{i}, \mathbf{a}))} f\left(y_{r}\right) .
$$

The proof is standard and will be omitted.

5.3.2. By $[\mathrm{Lu} 1,12.11]$ the endomorphisms $F_{i}$ satisfy the Serre relations

$$
\sum_{p=0}^{1-a_{i j}}(-1)^{p} F_{j}^{(p)} F_{i} F_{j}^{\left(1-a_{i j}-p\right)}=0
$$

for every $i \neq j$. A similar argument shows that 
Lemma 3. The endomorphisms $E_{i}$ satisfy the Serre relations

$$
\sum_{p=0}^{1-a_{i j}}(-1)^{p} E_{j}^{(p)} E_{i} E_{j}^{\left(1-a_{i j}-p\right)}=0
$$

for every $i \neq j$.

Proof. Let $f \in \widetilde{\mathcal{M}}_{\beta}^{\lambda}$ and $x \in \Lambda_{\beta-\alpha_{i}-\left(1-a_{i j}\right) \alpha_{j}}^{\lambda}$. By Lemma 2,

$$
\left(E_{j}^{(p)} E_{i} E_{j}^{\left(1-a_{i j}-p\right)} f\right)(x)=\int_{\mathfrak{f}} f\left(y_{3}\right)
$$

the integral being taken on the variety of flags

$$
\mathfrak{f}=\left(x \subset y_{1} \subset y_{2} \subset y_{3} \subset q_{\lambda}\right)
$$

with $y_{1} / x \cong s_{j}^{\oplus 1-a_{i j}-p}, y_{2} / y_{1} \cong s_{i}$ and $y_{3} / y_{2} \cong s_{j}^{\oplus p}$. This integral can be rewritten as

$$
\int_{y_{3}} f\left(y_{3}\right) \chi\left(\mathcal{F}\left[y_{3} ; p\right]\right)
$$

where the integral is now over all submodules $y_{3}$ of $q_{\lambda}$ of dimension $\beta$ containing $x$ and $\mathcal{F}\left[y_{3} ; p\right]$ is the variety of flags $\mathfrak{f}$ as above with fixed last step $y_{3}$. Now, by moding out the submodule $x$ at each step of the flag, we are reduced to the same situation as in [Lu1, 12.11], and the same argument allows to show that

$$
\sum_{p=0}^{1-a_{i j}} \chi\left(\mathcal{F}\left[y_{3} ; p\right]\right)=0
$$

which proves the Lemma.

5.3.3. Let $x \in \Lambda_{\beta}^{\lambda}$. Let $\varepsilon_{i}(x)$ denote the multiplicity of $s_{i}$ in the head of $x$. Let $\varphi_{i}(x)$ denote the multiplicity of $s_{i}$ in the socle of $q_{\lambda} / x$.

Lemma 4. Let $i, j \in I$ (not necessarily distinct). Let $y$ be a submodule of $q_{\lambda}$ containing $x$ and such that $y / x \cong s_{j}$. Then

$$
\varphi_{i}(y)-\varepsilon_{i}(y)=\varphi_{i}(x)-\varepsilon_{i}(x)-a_{i j}
$$


Proof. We have short exact sequences

$$
\begin{aligned}
& 0 \longrightarrow x \longrightarrow q_{\lambda} \longrightarrow q_{\lambda} / x \longrightarrow 0 \\
& 0 \longrightarrow y \longrightarrow q_{\lambda} \longrightarrow q_{\lambda} / y \longrightarrow 0 \\
& 0 \longrightarrow x \longrightarrow s_{j} \longrightarrow 0 \\
& 0 \longrightarrow s_{j} \longrightarrow q_{\lambda} / x \longrightarrow q_{\lambda} / y \longrightarrow 0
\end{aligned}
$$

Clearly, $\varepsilon_{i}(x)=\left|\operatorname{Hom}_{\Lambda}\left(x, s_{i}\right)\right|$, the dimension of $\operatorname{Hom}_{\Lambda}\left(x, s_{i}\right)$. Similarly $\varepsilon_{i}(y)=\left|\operatorname{Hom}_{\Lambda}\left(y, s_{i}\right)\right|, \varphi_{i}(x)=\left|\operatorname{Hom}_{\Lambda}\left(s_{i}, q_{\lambda} / x\right)\right|, \varphi_{i}(y)=\left|\operatorname{Hom}_{\Lambda}\left(s_{i}, q_{\lambda} / y\right)\right|$. Hence we have to show that

$$
\begin{aligned}
& \left|\operatorname{Hom}_{\Lambda}\left(x, s_{i}\right)\right|-\left|\operatorname{Hom}_{\Lambda}\left(y, s_{i}\right)\right| \\
& \quad=\left|\operatorname{Hom}_{\Lambda}\left(s_{i}, q_{\lambda} / x\right)\right|-\left|\operatorname{Hom}_{\Lambda}\left(s_{i}, q_{\lambda} / y\right)\right|-a_{i j} .
\end{aligned}
$$

In our proof, we will use a property of preprojective algebras proved in [CB, $\S 1]$, namely, for any finite-dimensional $\Lambda$-modules $m$ and $n$ there holds

$$
\left|\operatorname{Ext}_{\Lambda}^{1}(m, n)\right|=\left|\operatorname{Ext}_{\Lambda}^{1}(n, m)\right| .
$$

(a) If $i=j$ then $a_{i j}=2,\left|\operatorname{Hom}_{\Lambda}\left(s_{j}, s_{i}\right)\right|=1$ and $\left|\operatorname{Ext}_{\Lambda}^{1}\left(s_{j}, s_{i}\right)\right|=0$ since $\Gamma$ has no loops. Applying $\operatorname{Hom}_{\Lambda}\left(-, s_{i}\right)$ to $(9)$ we get the exact sequence

$$
0 \longrightarrow \operatorname{Hom}_{\Lambda}\left(s_{j}, s_{i}\right) \longrightarrow \operatorname{Hom}_{\Lambda}\left(y, s_{i}\right) \longrightarrow \operatorname{Hom}_{\Lambda}\left(x, s_{i}\right) \longrightarrow 0,
$$

hence

$$
\left|\operatorname{Hom}_{\Lambda}\left(x, s_{i}\right)\right|-\left|\operatorname{Hom}_{\Lambda}\left(y, s_{i}\right)\right|=-1 .
$$

Similarly applying $\operatorname{Hom}_{\Lambda}\left(s_{i},-\right)$ to $(10)$ we get an exact sequence

$$
0 \longrightarrow \operatorname{Hom}_{\Lambda}\left(s_{i}, s_{j}\right) \longrightarrow \operatorname{Hom}_{\Lambda}\left(s_{i}, q_{\lambda} / x\right) \longrightarrow \operatorname{Hom}_{\Lambda}\left(s_{i}, q_{\lambda} / y\right) \longrightarrow 0
$$

hence

$$
\left|\operatorname{Hom}_{\Lambda}\left(s_{i}, q_{\lambda} / x\right)\right|-\left|\operatorname{Hom}_{\Lambda}\left(s_{i}, q_{\lambda} / y\right)\right|=1,
$$

and (11) follows.

(b) If $i \neq j$, we have $\left|\operatorname{Hom}_{\Lambda}\left(s_{i}, s_{j}\right)\right|=0$ and $\left|\operatorname{Ext}_{\Lambda}^{1}\left(s_{i}, s_{j}\right)\right|=$ $\left|\operatorname{Ext}_{\Lambda}^{1}\left(s_{j}, s_{i}\right)\right|=-a_{i j}$. Applying $\operatorname{Hom}_{\Lambda}\left(s_{i},-\right)$ to $(9)$ we get an exact sequence

$$
0 \longrightarrow \operatorname{Hom}_{\Lambda}\left(s_{i}, x\right) \longrightarrow \operatorname{Hom}_{\Lambda}\left(s_{i}, y\right) \longrightarrow 0
$$

hence

$$
\left|\operatorname{Hom}_{\Lambda}\left(s_{i}, x\right)\right|-\left|\operatorname{Hom}_{\Lambda}\left(s_{i}, y\right)\right|=0 .
$$


Moreover, by [Bo, §1.1], $\left|\operatorname{Ext}_{\Lambda}^{2}\left(s_{i}, s_{j}\right)\right|=0$ because there are no relations from $i$ to $j$ in the defining relations of $\Lambda$. (Note that the proof of this result in [Bo] only requires that $I \subseteq J^{2}$ (here we use the notation of [Bo]). One does not need the additional assumption $J^{n} \subseteq I$ for some $n$. Compare also the discussion in $[\mathrm{BK}]$.)

Since $q_{\lambda}$ is injective $\left|\operatorname{Ext}_{\Lambda}^{1}\left(s_{i}, q_{\lambda}\right)\right|=0$, thus applying $\operatorname{Hom}_{\Lambda}\left(s_{i},-\right)$ to (7) we get an exact sequence

$$
\begin{aligned}
0 & \longrightarrow \operatorname{Hom}_{\Lambda}\left(s_{i}, x\right) \longrightarrow \operatorname{Hom}_{\Lambda}\left(s_{i}, q_{\lambda}\right) \longrightarrow \operatorname{Hom}_{\Lambda}\left(s_{i}, q_{\lambda} / x\right) \\
& \longrightarrow \operatorname{Ext}_{\Lambda}^{1}\left(s_{i}, x\right) \longrightarrow 0
\end{aligned}
$$

hence

$$
\left|\operatorname{Hom}_{\Lambda}\left(s_{i}, x\right)\right|-\left|\operatorname{Hom}_{\Lambda}\left(s_{i}, q_{\lambda}\right)\right|+\left|\operatorname{Hom}_{\Lambda}\left(s_{i}, q_{\lambda} / x\right)\right|-\left|\operatorname{Ext}_{\Lambda}^{1}\left(s_{i}, x\right)\right|=0 .
$$

Similarly, applying $\operatorname{Hom}_{\Lambda}\left(s_{i},-\right)$ to $(8)$ we get

(15) $\left|\operatorname{Hom}_{\Lambda}\left(s_{i}, y\right)\right|-\left|\operatorname{Hom}_{\Lambda}\left(s_{i}, q_{\lambda}\right)\right|+\left|\operatorname{Hom}_{\Lambda}\left(s_{i}, q_{\lambda} / y\right)\right|-\left|\operatorname{Ext}_{\Lambda}^{1}\left(s_{i}, y\right)\right|=0$.

Subtracting (14) from (15) and taking into account (12) and (13) we obtain

$$
\left|\operatorname{Ext}_{\Lambda}^{1}\left(x, s_{i}\right)\right|-\left|\operatorname{Ext}_{\Lambda}^{1}\left(y, s_{i}\right)\right|=\left|\operatorname{Hom}_{\Lambda}\left(s_{i}, q_{\lambda} / x\right)\right|-\left|\operatorname{Hom}_{\Lambda}\left(s_{i}, q_{\lambda} / y\right)\right| .
$$

Now applying $\operatorname{Hom}_{\Lambda}\left(-, s_{i}\right)$ to $(9)$ we get the long exact sequence

$$
\begin{aligned}
0 & \longrightarrow \operatorname{Hom}_{\Lambda}\left(y, s_{i}\right) \longrightarrow \operatorname{Hom}_{\Lambda}\left(x, s_{i}\right) \longrightarrow \operatorname{Ext}_{\Lambda}^{1}\left(s_{j}, s_{i}\right) \\
& \longrightarrow \operatorname{Ext}_{\Lambda}^{1}\left(y, s_{i}\right) \longrightarrow \operatorname{Ext}_{\Lambda}^{1}\left(x, s_{i}\right) \longrightarrow 0,
\end{aligned}
$$

hence

$$
\left|\operatorname{Hom}_{\Lambda}\left(y, s_{i}\right)\right|-\left|\operatorname{Hom}_{\Lambda}\left(x, s_{i}\right)\right|-a_{i j}-\left|\operatorname{Ext}_{\Lambda}^{1}\left(y, s_{i}\right)\right|+\left|\operatorname{Ext}_{\Lambda}^{1}\left(x, s_{i}\right)\right|=0,
$$

thus, taking into account (16), we have proved (11).

LEMMA 5. With the same notation we have

$$
\varphi_{i}(x)-\varepsilon_{i}(x)=\left(\lambda-\beta ; \alpha_{i}\right) .
$$

Proof. We use an induction on the height of $\beta$. If $\beta=0$ then $x$ is the zero module and $\varepsilon_{i}(x)=0$. On the other hand $q_{\lambda} / x=q_{\lambda}$ and $\varphi_{i}(x)=\left(\lambda ; \alpha_{i}\right)$ by definition of $q_{\lambda}$. Now assume that the lemma holds for $x \in \Lambda_{\beta}^{\lambda}$ and let $y \in \Lambda_{\beta+\alpha_{j}}^{\lambda}$ be a submodule of $q_{\lambda}$ containing $x$. Using Lemma 4 we get that

$$
\varphi_{i}(y)-\varepsilon_{i}(y)=\left(\lambda-\beta ; \alpha_{i}\right)-a_{i j}=\left(\lambda-\beta-\alpha_{j} ; \alpha_{i}\right),
$$

as required, and the lemma follows. 
Lemma 6. Let $f \in \widetilde{\mathcal{M}}_{\beta}^{\lambda}$. We have

$$
\left(E_{i} F_{j}-F_{j} E_{i}\right)(f)=\delta_{i j}\left(\lambda-\beta ; \alpha_{i}\right) f
$$

Proof. Let $x \in \Lambda_{\beta-\alpha_{i}+\alpha_{j}}^{\lambda}$. By definition of $E_{i}$ and $F_{j}$ we have

$$
\left(E_{i} F_{j} f\right)(x)=\int_{\mathfrak{p} \in \mathfrak{P}} f(y)
$$

where $\mathfrak{P}$ denotes the variety of pairs $\mathfrak{p}=(u, y)$ of submodules of $q_{\lambda}$ with $x \subset u, y \subset u, u / x \cong s_{i}$ and $u / y \cong s_{j}$. Similarly,

$$
\left(F_{j} E_{i} f\right)(x)=\int_{\mathfrak{q} \in \mathfrak{Q}} f(y)
$$

where $\mathfrak{Q}$ denotes the variety of pairs $\mathfrak{q}=(v, y)$ of submodules of $q_{\lambda}$ with $v \subset x, v \subset y, x / v \cong s_{j}$ and $y / v \cong s_{i}$.

Consider a submodule $y$ such that there exists in $\mathfrak{P}($ resp. in $\mathfrak{Q})$ at least one pair of the form $(u, y)$ (resp. $(v, y)$ ). Clearly, the subspaces carrying the submodules $x$ and $y$ have the same dimension $d$ and their intersection has dimension at least $d-1$. If this intersection has dimension exactly $d-1$ then there is a unique pair $(u, y)($ resp. $(v, y))$, namely $(x+y, y)(\operatorname{resp} .(x \cap y, y))$. This means that

$$
\int_{\mathfrak{p} \in \mathfrak{P} ; y \neq x} f(y)=\int_{\mathfrak{q} \in \mathfrak{Q} ; y \neq x} f(y) .
$$

In particular, since when $i \neq j$ we cannot have $y=x$, it follows that

$$
\left(E_{i} F_{j}-F_{j} E_{i}\right)(f)=0, \quad(i \neq j)
$$

On the other hand if $i=j$ we have

$$
\left(\left(E_{i} F_{i}-F_{i} E_{i}\right)(f)\right)(x)=f(x)\left(\chi\left(\mathfrak{P}^{\prime}\right)-\chi\left(\mathfrak{Q}^{\prime}\right)\right)
$$

where $\mathfrak{P}^{\prime}$ is the variety of submodules $u$ of $q_{\lambda}$ containing $x$ such that $u / x \cong$ $s_{i}$, and $\mathfrak{Q}^{\prime}$ is the variety of submodules $v$ of $x$ such that $x / v \cong s_{i}$. Clearly we have $\chi\left(\mathfrak{Q}^{\prime}\right)=\varepsilon_{i}(x)$ and $\chi\left(\mathfrak{P}^{\prime}\right)=\varphi_{i}(x)$. The result then follows from Lemma 5. 
5.3.4. The following relations for the endomorphisms $E_{i}, F_{i}, H_{i}$ of $\widetilde{\mathcal{M}}^{\lambda}$ are easily checked

$$
\left[H_{i}, H_{j}\right]=0, \quad\left[H_{i}, E_{j}\right]=a_{i j} E_{j}, \quad\left[H_{i}, F_{j}\right]=-a_{i j} F_{j} .
$$

The verification is left to the reader. Hence, using Lemmas 3 and 6 , we have proved that the assignments $e_{i} \mapsto E_{i}, f_{i} \mapsto F_{i}, h_{i} \mapsto H_{i}$, give a representation of $\mathfrak{g}$ on $\widetilde{\mathcal{M}}^{\lambda}$.

LEMMA 7. The endomorphisms $E_{i}, F_{i}, H_{i}$ leave stable the subspace $\mathcal{M}^{\lambda}$.

Proof. It is obvious for $H_{i}$, and it follows from the definition of $\mathcal{M}^{\lambda}$ for $F_{i}$. It remains to prove that if $f \in \mathcal{M}_{\beta}^{\lambda}$ then $E_{i} f \in \mathcal{M}_{\beta-\alpha_{i}}^{\lambda}$. We shall use induction on the height of $\beta$. We can assume that $f$ is of the form $F_{j} g$ for some $g \in \mathcal{M}_{\beta-\alpha_{j}}^{\lambda}$. By induction we can also assume that $E_{i} g \in \mathcal{M}_{\beta-\alpha_{i}-\alpha_{j}}^{\lambda}$. We have

$$
E_{i} f=E_{i} F_{j} g=F_{j} E_{i} g+\delta_{i j}\left(\lambda-\beta+\alpha_{j} ; \alpha_{i}\right) g
$$

and the right-hand side clearly belongs to $\mathcal{M}_{\beta-\alpha_{i}}^{\lambda}$.

LEMMA 8. The representation of $\mathfrak{g}$ carried by $\mathcal{M}^{\lambda}$ is isomorphic to $L(\lambda)$.

Proof. For all $f \in \mathcal{M}_{\beta}$ and all $x \in \Lambda_{\beta+\left(a_{i}+1\right) \alpha_{i}}^{\lambda}$ we have $f * \mathbf{1}_{i}^{*\left(a_{i}+1\right)}(x)=$ 0 . Indeed, by definition of $\Lambda^{\lambda}$ the socle of $x$ contains $s_{i}$ with multiplicity at most $a_{i}$. Therefore the left ideal of $\mathcal{M}$ generated by the functions $\mathbf{1}_{i}^{*\left(a_{i}+1\right)}$ is mapped to zero by the linear map $\mathcal{M} \rightarrow \mathcal{M}^{\lambda}$ sending a function $f$ on $\Lambda_{\beta}$ to its restriction to $\Lambda_{\beta}^{\lambda}$. It follows that for all $\beta$ the dimension of $\mathcal{M}_{\beta}^{\lambda}$ is at most the dimension of the $(\lambda-\beta)$-weight space of $L(\lambda)$.

On the other hand, the function $\mathbf{1}_{0}$ mapping the zero $\Lambda$-module to 1 is a highest weight vector of $\mathcal{M}^{\lambda}$ of weight $\lambda$. Hence $\mathbf{1}_{0} \in \mathcal{M}^{\lambda}$ generates a quotient of the Verma module $M(\lambda)$, and since $L(\lambda)$ is the smallest quotient of $M(\lambda)$ we must have $\mathcal{M}^{\lambda}=L(\lambda)$.

This finishes the proof of Theorem 1 . 


\section{§6. Geometric realization of Verma modules}

6.1. Let $\beta \in Q_{+}$and $x \in \Lambda_{\beta-\alpha_{i}}$. Let $q=\bigoplus_{i \in I} q_{i}^{\oplus a_{i}}$ be the injective hull of $x$. For every $\nu \in P_{+}$such that $\left(\nu ; \alpha_{i}\right) \geqslant a_{i}$ the injective module $q_{\nu}$ contains a submodule isomorphic to $x$. Hence, for such a weight $\nu$ and for any $f \in \mathcal{M}_{\beta}$, the integral

$$
\int_{y \in \mathcal{G}(x, \nu, i)} f(y)
$$

is well-defined.

Proposition 1. Let $\lambda \in P$ and choose $\nu \in P_{+}$such that $\left(\nu ; \alpha_{i}\right) \geqslant a_{i}$ for all $i \in I$. The number

$$
\int_{y \in \mathcal{G}(x, \nu, i)} f(y)-\left(\nu-\lambda ; \alpha_{i}\right) f\left(x \oplus s_{i}\right)
$$

does not depend on the choice of $\nu$. Denote this number by $\left(E_{i}^{\lambda} f\right)(x)$. Then, the function

$$
E_{i}^{\lambda} f: x \longmapsto\left(E_{i}^{\lambda} f\right)(x)
$$

belongs to $\mathcal{M}_{\beta-\alpha_{i}}$.

Denote by $E_{i}^{\lambda}$ the endomorphism of $\mathcal{M}$ mapping $f \in \mathcal{M}_{\beta}$ to $E_{i}^{\lambda} f$. Notice that Formula (5), which is nothing but (3), also defines an endomorphism of $\mathcal{M}$ independent of $\lambda$ which we again denote by $F_{i}$. Finally Formula (6) makes sense for any $\lambda$, not necessarily dominant, and any $f \in \mathcal{M}_{\beta}$. This gives an endomorphism of $\mathcal{M}$ that we shall denote by $H_{i}^{\lambda}$.

Theorem 2. The assignments $e_{i} \mapsto E_{i}^{\lambda}, f_{i} \mapsto F_{i}, h_{i} \mapsto H_{i}^{\lambda}$, give a representation of $\mathfrak{g}$ on $\mathcal{M}$ isomorphic to the Verma module $M(\lambda)$.

The rest of this section is devoted to the proofs of Proposition 1 and Theorem 2 .

6.2. Denote by $e_{i}^{\lambda}$ the endomorphism of the Verma module $M(\lambda)$ implementing the action of the Chevalley generator $e_{i}$. Let $\mathcal{E}_{i}^{\lambda}$ denote the endomorphism of $U\left(\mathfrak{n}_{-}\right)$obtained by transporting $e_{i}^{\lambda}$ via the natural identification $M(\lambda) \cong U\left(\mathfrak{n}_{-}\right)$. Let $\Delta$ be the comultiplication of $U\left(\mathfrak{n}_{-}\right)$.

Lemma 9. For $\lambda, \mu \in P$ and $u \in U\left(\mathfrak{n}_{-}\right)$we have

$$
\Delta\left(\mathcal{E}_{i}^{\lambda+\mu} u\right)=\left(\mathcal{E}_{i}^{\lambda} \otimes 1+1 \otimes \mathcal{E}_{i}^{\mu}\right) \Delta u .
$$


Proof. By linearity it is enough to prove this for $u$ of the form $u=$ $f_{i_{1}} \cdots f_{i_{r}}$. A simple calculation in $U(\mathfrak{g})$ shows that

$$
\begin{aligned}
e_{i} f_{i_{1}} \cdots f_{i_{r}}= & f_{i_{1}} \cdots f_{i_{r}} e_{i}+\sum_{k=1}^{r} \delta_{i i_{k}} f_{i_{1}} \cdots f_{i_{k-1}} h_{i} f_{i_{k+1}} \cdots f_{i_{r}} \\
=f_{i_{1}} \cdots f_{i_{r}} e_{i}+\sum_{k=1}^{r} \delta_{i i_{k}}\left(f_{i_{1}} \cdots f_{i_{k-1}} f_{i_{k+1}} \cdots f_{i_{r}} h_{i}\right. & \\
& \left.\quad-\left(\sum_{s=k+1}^{r} a_{i i_{s}}\right) f_{i_{1}} \cdots f_{i_{k-1}} f_{i_{k+1}} \cdots f_{i_{r}}\right)
\end{aligned}
$$

It follows that, for $\nu \in P$,

$$
\mathcal{E}_{i}^{\nu}\left(f_{i_{1}} \cdots f_{i_{r}}\right)=\sum_{k=1}^{r} \delta_{i i_{k}}\left(\left(\nu ; \alpha_{i}\right)-\sum_{s=k+1}^{r} a_{i i_{s}}\right) f_{i_{1}} \cdots f_{i_{k-1}} f_{i_{k+1}} \cdots f_{i_{r}} .
$$

Now, using that $\Delta$ is the algebra homomorphism defined by $\Delta\left(f_{i}\right)=f_{i} \otimes$ $1+1 \otimes f_{i}$, one can finish the proof of the lemma. Details are omitted.

6.3. We endow $U\left(\mathfrak{n}_{-}\right)$with the $Q_{+}$-grading given by $\operatorname{deg}\left(f_{i}\right)=\alpha_{i}$. Let $u$ be a homogeneous element of $U\left(\mathfrak{n}_{-}\right)$. Write $\Delta u=u \otimes 1+u^{(i)} \otimes f_{i}+A$, where $A$ is a sum of homogeneous terms of the form $u^{\prime} \otimes u^{\prime \prime}$ with $\operatorname{deg}\left(u^{\prime \prime}\right) \neq \alpha_{i}$. This defines $u^{(i)}$ unambiguously.

Lemma 10. For $\lambda, \mu \in P$ we have

$$
\mathcal{E}_{i}^{\lambda+\mu} u=\mathcal{E}_{i}^{\lambda} u+\left(\mu ; \alpha_{i}\right) u^{(i)}
$$

Proof. We calculate in two ways the unique term of the form $E \otimes 1$ in $\Delta\left(\mathcal{E}_{i}^{\lambda+\mu} u\right)$. On the one hand, we have obviously $E \otimes 1=\mathcal{E}_{i}^{\lambda+\mu} u \otimes 1$. On the other hand, using Lemma 9, we have

$$
E \otimes 1=\mathcal{E}_{i}^{\lambda} u \otimes 1+\left(1 \otimes \mathcal{E}_{i}^{\mu}\right)\left(u^{(i)} \otimes f_{i}\right)=\mathcal{E}_{i}^{\lambda} u \otimes 1+\left(\mu ; \alpha_{i}\right) u^{(i)} \otimes 1
$$

Therefore,

$$
E=\mathcal{E}_{i}^{\lambda+\mu} u=\mathcal{E}_{i}^{\lambda} u+\left(\mu ; \alpha_{i}\right) u^{(i)}
$$


6.4. Now let us return to the geometric realization $\mathcal{M}$ of $U\left(\mathfrak{n}_{-}\right)$. Let $E_{i}^{\lambda}$ denote the endomorphism of $\mathcal{M}$ obtained by transporting $e_{i}^{\lambda}$ via the identification $M(\lambda) \cong \mathcal{M}$.

Lemma 11. Let $\lambda \in P_{+}, f \in \mathcal{M}_{\beta}$ and $x \in \Lambda_{\beta-\alpha_{i}}^{\lambda}$. Then

$$
\left(E_{i}^{\lambda} f\right)(x)=\int_{y \in \mathcal{G}(x, \lambda, i)} f(y) .
$$

Proof. Let $r_{\lambda}: \mathcal{M} \rightarrow \mathcal{M}^{\lambda}$ be the linear map sending $f \in \mathcal{M}_{\beta}$ to its restriction to $\Lambda_{\beta}^{\lambda}$. By Theorem 1, this is a homomorphism of $U\left(\mathfrak{n}_{-}\right)$modules mapping the highest weight vector of $\mathcal{M} \cong M(\lambda)$ to the highest weight vector of $\mathcal{M}^{\lambda} \cong L(\lambda)$. It follows that $r_{\lambda}$ is in fact a homomorphism of $U(\mathfrak{g})$-modules, hence the restriction of $E_{i}^{\lambda} f$ to $\Lambda_{\beta-\alpha_{i}}^{\lambda}$ is given by Formula (4) of Section 5 .

Let again $\lambda \in P$ be arbitrary, and pick $f \in \mathcal{M}_{\beta}$. It follows from Lemma 10 that for any $\mu \in P$

$$
E_{i}^{\lambda+\mu} f-\left(\mu ; \alpha_{i}\right) f^{(i)}=E_{i}^{\lambda} f
$$

Let $x \in \Lambda_{\beta-\alpha_{i}}$. Choose $\nu=\lambda+\mu$ sufficiently dominant so that $x$ is isomorphic to a submodule of $q_{\nu}$. Then by Lemma 11, we have

$$
\left(E_{i}^{\nu} f\right)(x)=\int_{y \in \mathcal{G}(x, \nu, i)} f(y) .
$$

On the other hand, by the geometric description of $\Delta$ given in [GLS, §6.1], if we write

$$
\Delta f=f \otimes 1+f^{(i)} \otimes \mathbf{1}_{i}+A
$$

where $A$ is a sum of homogeneous terms of the form $f^{\prime} \otimes f^{\prime \prime}$ with $\operatorname{deg}\left(f^{\prime \prime}\right) \neq$ $\alpha_{i}$, we have that $f^{(i)}$ is the function on $\Lambda_{\beta-\alpha_{i}}$ given by $f^{(i)}(x)=f\left(x \oplus s_{i}\right)$. Hence we obtain that for $x \in \Lambda_{\beta-\alpha_{i}}$

$$
\left(E_{i}^{\lambda} f\right)(x)=\int_{y \in \mathcal{G}(x, \nu, i)} f(y)-\left(\nu-\lambda ; \alpha_{i}\right) f\left(x \oplus s_{i}\right) .
$$

This proves both Proposition 1 and Theorem 2. 
6.5. Let $\lambda \in P_{+}$. We note the following consequence of Lemma 11 .

Proposition 2. Let $\lambda \in P_{+}$. The linear map $r_{\lambda}: \mathcal{M} \rightarrow \mathcal{M}^{\lambda}$ sending $f \in \mathcal{M}_{\beta}$ to its restriction to $\Lambda_{\beta}^{\lambda}$ is the geometric realization of the homomorphism of $\mathfrak{g}$-modules $M(\lambda) \rightarrow L(\lambda)$.

\section{$\S 7$. Dual Verma modules}

7.1. Let $S$ be the anti-automorphism of $U(\mathfrak{g})$ defined by

$$
S\left(e_{i}\right)=f_{i}, \quad S\left(f_{i}\right)=e_{i}, \quad S\left(h_{i}\right)=h_{i}, \quad(i \in I) .
$$

Recall that, given a left $U(\mathfrak{g})$-module $M$, the dual module $M^{*}$ is defined by

$$
(u \varphi)(m)=\varphi(S(u) m), \quad\left(u \in U(\mathfrak{g}), m \in M, \varphi \in M^{*}\right) .
$$

This is also a left module. If $M$ is an infinite-dimensional module with finite-dimensional weight spaces $M_{\nu}$, we take for $M^{*}$ the graded dual $M^{*}=$ $\bigoplus_{\nu \in P} M_{\nu}^{*}$

For $\lambda \in P$ we have $L(\lambda)^{*} \cong L(\lambda)$, hence the quotient map $M(\lambda) \rightarrow L(\lambda)$ gives by duality an embedding $L(\lambda) \rightarrow M(\lambda)^{*}$ of $U(\mathfrak{g})$-modules.

7.2. Let $\mathcal{M}^{*}=\bigoplus_{\beta \in Q_{+}} \mathcal{M}_{\beta}^{*}$ denote the vector space graded dual of $\mathcal{M}$. For $x \in \Lambda_{\beta}$, we denote by $\delta_{x}$ the delta function given by

$$
\delta_{x}(f)=f(x), \quad\left(f \in \mathcal{M}_{\beta}\right) .
$$

Note that the map $\delta: x \mapsto \delta_{x}$ is a constructible map from $\Lambda_{\beta}$ to $\mathcal{M}_{\beta}^{*}$. Indeed the preimage of $\delta_{x}$ is the intersection of the constructible subsets

$$
\begin{aligned}
\mathcal{M}_{\left(i_{1}, \ldots, i_{r}\right)}=\left\{y \in \Lambda_{\beta} \mid\left(\mathbf{1}_{i_{1}} * \cdots * \mathbf{1}_{i_{r}}\right)(y)=\left(\mathbf{1}_{i_{1}} * \cdots * \mathbf{1}_{i_{r}}\right)(x)\right\} \\
\left(\alpha_{i_{1}}+\cdots+\alpha_{i_{r}}=\beta\right) .
\end{aligned}
$$

7.3. We can now dualize the results of Sections 5 and 6 as follows. For $\lambda \in P$ and $x \in \Lambda_{\beta}$ put

$$
\begin{aligned}
\left(E_{i}^{*}\right)\left(\delta_{x}\right) & =\int_{y \in \mathcal{G}(i, x)} \delta_{y}, \\
\left(F_{i}^{\lambda *}\right)\left(\delta_{x}\right) & =\int_{y \in \mathcal{G}(x, \nu, i)} \delta_{y}-\left(\nu-\lambda ; \alpha_{i}\right) \delta_{x \oplus s_{i}}, \\
\left(H_{i}^{\lambda *}\right)\left(\delta_{x}\right) & =\left(\lambda-\beta ; \alpha_{i}\right) \delta_{x},
\end{aligned}
$$

where in (19) the weight $\nu \in P_{+}$is such that $x$ is isomorphic to a submodule of $q_{\nu}$. The following theorem then follows immediately from Theorems 1 and 2 . 
TheOREM 3. (i) The formulas above define endomorphisms $E_{i}^{*}, F_{i}^{\lambda *}$, $H_{i}^{\lambda *}$ of $\mathcal{M}^{*}$, and the assignments $e_{i} \mapsto E_{i}^{*}, f_{i} \mapsto F_{i}^{\lambda *}, h_{i} \mapsto H_{i}^{\lambda *}$, give a representation of $\mathfrak{g}$ on $\mathcal{M}^{*}$ isomorphic to the dual Verma module $M(\lambda)^{*}$.

(ii) If $\lambda \in P_{+}$, the subspace $\mathcal{M}^{\lambda *}$ of $\mathcal{M}^{*}$ spanned by the delta functions $\delta_{x}$ of the finite-dimensional nilpotent submodules $x$ of $q_{\lambda}$ carries the irreducible submodule $L(\lambda)$. For such a module $x$, Formula (19) simplifies as follows

$$
\left(F_{i}^{\lambda *}\right)\left(\delta_{x}\right)=\int_{y \in \mathcal{G}(x, \lambda, i)} \delta_{y} .
$$

Example 1. Let $\mathfrak{g}$ be of type $A_{2}$. Take $\lambda=\varpi_{1}+\varpi_{2}$, where $\varpi_{i}$ is the fundamental weight corresponding to $i \in I$. Thus $L(\lambda)$ is isomorphic to the 8-dimensional adjoint representation of $\mathfrak{g}=\mathfrak{s l}_{3}$.

A $\Lambda$-module $x$ consists of a pair of linear maps $x_{21}: V_{1} \rightarrow V_{2}$ and $x_{12}: V_{2} \rightarrow V_{1}$ such that $x_{12} x_{21}=x_{21} x_{12}=0$. The injective $\Lambda$-module $q=q_{\lambda}$ has the following form:

$$
q=\left(\begin{array}{l}
u_{1} \longrightarrow u_{2} \\
v_{1} \longleftarrow v_{2}
\end{array}\right)
$$

This diagram means that $\left(u_{1}, v_{1}\right)$ is a basis of $V_{1}$, that $\left(u_{2}, v_{2}\right)$ is a basis of $V_{2}$, and that

$$
q_{21}\left(u_{1}\right)=u_{2}, \quad q_{21}\left(v_{1}\right)=0, \quad q_{12}\left(v_{2}\right)=v_{1}, \quad q_{12}\left(u_{2}\right)=0 .
$$

Using the same type of notation, we can exhibit the following submodules of $q$ :

$$
\begin{aligned}
& x_{1}=\left(v_{1}\right), \quad x_{2}=\left(u_{2}\right), \quad x_{3}=\left(\begin{array}{ll}
v_{1} & u_{2}
\end{array}\right), \quad x_{4}=\left(u_{1} \longrightarrow u_{2}\right), \\
& x_{5}=\left(v_{1} \longleftarrow v_{2}\right), \quad x_{6}=\left(\begin{array}{l}
u_{1} \longrightarrow u_{2} \\
v_{1}
\end{array}\right), \quad x_{7}=\left(\begin{array}{r}
u_{2} \\
v_{1} \longleftarrow v_{2}
\end{array}\right) \text {. }
\end{aligned}
$$

This is not an exhaustive list. For example, $x_{4}^{\prime}=\left(\left(u_{1}+v_{1}\right) \longrightarrow u_{2}\right)$ is another submodule, isomorphic to $x_{4}$. Denoting by $\mathbf{0}$ the zero submodule, we see that $\delta_{\mathbf{0}}$ is the highest weight vector of $L(\lambda) \subset M(\lambda)^{*}$. Next, writing for simplicity $\delta_{i}$ instead of $\delta_{x_{i}}$ and $F_{i}$ instead of $F_{i}^{\lambda}$, Theorem 3 (ii) gives the following formulas for the action of the $F_{i}$ 's on $L(\lambda)$.

$$
\begin{gathered}
F_{1} \delta_{\mathbf{0}}=\delta_{1}, \quad F_{2} \delta_{\mathbf{0}}=\delta_{2}, \quad F_{1} \delta_{2}=\delta_{3}+\delta_{4}, \quad F_{2} \delta_{1}=\delta_{3}+\delta_{5}, \\
F_{1} \delta_{3}=F_{1} \delta_{4}=\delta_{6}, \quad F_{2} \delta_{3}=F_{2} \delta_{5}=\delta_{7}, \\
F_{2} \delta_{3}=F_{1} \delta_{6}=\delta_{q}, \quad F_{1} \delta_{q}=F_{2} \delta_{q}=0 .
\end{gathered}
$$


Now consider the $\Lambda$-module $x=s_{1} \oplus s_{1}$. Since $x$ is not isomorphic to a submodule of $q_{\lambda}$, the vector $\delta_{x}$ does not belong to $L(\lambda)$. Let us calculate $F_{i} \delta_{x}(i=1,2)$ by means of Formula (19). We can take $\nu=2 \varpi_{1}$. The injective $\Lambda$-module $q_{\nu}$ has the following form:

$$
q_{\nu}=\left(\begin{array}{c}
w_{1} \longleftarrow w_{2} \\
v_{1} \longleftarrow v_{2}
\end{array}\right)
$$

It is easy to see that the variety $\mathcal{G}(x, \nu, 2)$ is isomorphic to a projective line $\mathbb{P}_{1}$, and that all points on this line are isomorphic to

$$
y=\left(\begin{array}{l}
w_{1} \\
v_{1} \longleftarrow v_{2}
\end{array}\right)
$$

as $\Lambda$-modules. Hence,

$$
F_{2} \delta_{x}=\chi\left(\mathbb{P}_{1}\right) \delta_{y}-\left(\nu-\lambda ; \alpha_{2}\right) \delta_{x \oplus s_{2}}=2 \delta_{y}+\delta_{s_{1} \oplus s_{1} \oplus s_{2}}
$$

On the other hand, $\mathcal{G}(x, \nu, 1)=\emptyset$, so that

$$
F_{1} \delta_{x}=-\left(\nu-\lambda ; \alpha_{1}\right) \delta_{x \oplus s_{1}}=-\delta_{s_{1} \oplus s_{1} \oplus s_{1}}
$$

\section{REFERENCES}

[Bo] K. Bongartz, Algebras and quadratic forms, J. London Math. Soc., 28 (1983), 461-469.

[BK] M. C. R. Butler and A. D. King, Minimal resolutions of algebras, J. Algebra, 212 (1999), 323-362.

[CB] W. Crawley-Boevey, On the exceptional fibres of Kleinian singularities, Amer. J. Math., 122 (2000), 1027-1037.

[GLS] C. Geiss, B. Leclerc and J. Schröer, Semicanonical bases and preprojective algebras, Ann. Scient. Éc. Norm. Sup., 38 (2005), 193-253.

[GP] I. M. Gelfand and V. A. Ponomarev, Model algebras and representations of graphs, Funct. Anal. Appl., 13 (1980), 157-166.

[Lu1] G. Lusztig, Quivers, perverse sheaves, and quantized enveloping algebras, J. Amer. Math. Soc., 4 (1991), 365-421.

[Lu2] G. Lusztig, Semicanonical bases arising from enveloping algebras, Adv. Math., 151 (2000), 129-139.

[Lu3] G. Lusztig, Remarks on quiver varieties, Duke Math. J., 105 (2000), 239-265.

[Lu4] G. Lusztig, Constructible functions on varieties attached to quivers, Studies in memory of Issai Schur, Progress in Mathematics 210, Birkhäuser (2003), pp. 177-223. 
[Na] H. Nakajima, Instantons on ALE spaces, quiver varieties, and Kac-Moody algebras, Duke Math. J., 76 (1994), 365-416.

[Ri] C. M. Ringel, The preprojective algebra of a quiver, Algebras and modules II (Geiranger, 1966), CMS Conf. Proc. 24, AMS (1998), pp. 467-480.

Christof Geiss

Instituto de Matemáticas, UNAM

Ciudad Universitaria

04510 Mexico D.F.

Mexico

christof@math . unam . mx

Bernard Leclerc

LMNO, Université de Caen

14032 Caen cedex

France

leclerc@math.unicaen.fr

Jan Schröer

Department of Pure Mathematics

University of Leeds

Leeds LS2 9JT

England

jschroer@maths. leeds.ac.uk 